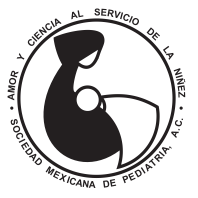

\title{
Acalasia cricofaríngea: a propósito de un caso
}

\author{
Marta del Pino-García,, ${ }^{1, *}$ Mercedes Murray-Hurtado, ${ }^{2}$ \\ Juan Antonio Hernández-Ponce, ${ }^{3}$ María Teresa Rodrigo-Bello ${ }^{4}$ \\ ${ }^{1}$ Residente en Pediatría y Áreas Especificas. Complejo Hospitalario Universitario de Canarias, \\ Tenerife; ${ }^{2}$ Especialista en Pediatría y Áreas Especificas. Complejo Hospitalario Universitario de Canarias, Tenerife; \\ ${ }^{3}$ Especialista en Radiodiagnóstico. Complejo Hospitalario Universitario Insular Materno-Infantil, Gran Canaria; \\ ${ }^{4}$ Especialista en Radiodiagnóstico. Complejo Hospitalario Universitario de Canarias, Tenerife, España.
}

\begin{abstract}
RESUMEN
Introducción: La acalasia cricofaríngea (AC) es una causa rara de disfagia en pediatría, con origen multifactorial. Sus manifestaciones clínicas son diversas e inespecíficas, lo que dificulta el diagnóstico. Los estudios de imagen contrastados como el tránsito esofagogastroduodenal (TEGD) constituyen el estándar de referencia para llegar al diagnóstico. Caso clínico: Lactante de un mes de vida con fallo de medro. A la alimentación, succiona con avidez, pero inmediatamente comienza a acumular leche en la boca, llegando a expulsarla por la nariz y, en ocasiones, accesos de tos. En la exploración física destaca un peso en percentil 2. En esofagograma se observó disminución del calibre del esófago superior con dilatación proximal, regurgitación nasal y paso de contraste a la vía respiratoria. Se inicia nutrición por sonda nasogástrica y, a los dos meses de vida, se realizan dos dilataciones neumáticas sin éxito. Posteriormente, a los cinco meses se efectúa cricomiotomía con resolución completa de la sintomatología. Conclusiones: La AC es una anomalía congénita poco frecuente, pero se puede diagnosticar mediante TEGD; en niños el tratamiento no está claramente definido.
\end{abstract}

Palabras clave: Acalasia cricofaríngea, disfagia, lactante, cricomiotomía.

\begin{abstract}
Introduction: Cricopharyngeal achalasia (CA) is a rare cause of dysphagia in children. Clinical manifestations are diverse and nonspecific, which leads to delay in the diagnosis. Radiological studies, such as esophagogastroduodenal transit, constitute the gold standar to achieve diagnosis. Clinical case: Female infant of a month of life with failure to thrive. At feeding, she sucks greedily, but immediately begins to accumulate milk in the mouth, getting to expel it through the nose and, sometimes, she coughs. On physical examination, her weight was found in percentile 2. In esophagogram, a decrease in the caliber of the upper esophagus was observed with proximal dilation, nasal regurgitation and the contrast medium passing into the respiratory tract. Nasogastric tube feeding was started and, after two months of life, two pneumatic dilations were performed without success. Subsequently, after five months, crichiotomy was performed, which resulted in a complete resolution of the symptomatology. Conclusions: CA is a rare congenital anomaly, but it can be diagnosed by esophagogastroduodenal transit. In children, treatment is not clearly defined.
\end{abstract}

Keywords: Cricopharyngeal achalasia, dysphagia, infant, cricomiotomy.

\footnotetext{
*Correspondencia: MPG, martadelpinogarcia@gmail.com Conflicto de intereses: Los autores declaran que no tienen. Citar como: Del Pino-García M, Murray-Hurtado M, HernándezPonce JA, Rodrigo-Bello MT. Acalasia cricofaríngea: a propósito de un caso. Rev Mex Pediatr 2019; 86(6):234-237. doi: 10.35366/91875 [Cricopharyngeal achalasia: a case report]
}

\section{INTRODUCCIÓN}

En la edad pediátrica, las alteraciones de la deglución raramente se presentan como problemas aislados, sino más bien parten de otras enfermedades. Algunas de estas entidades pueden llegar a ser mortales en la medida que comprometan la vía aérea. ${ }^{1}$

La disfagia puede ocurrir por un trastorno de origen orofaríngeo o esofágico. ${ }^{2}$ Normalmente, tras la 
formación del bolo alimentario y su paso a través de la faringe, la fisiología normal de la deglución requiere la apertura del esfínter esofágico superior (EES), para permitir el paso del bolo desde la faringe hacia el esófago. Esta apertura de la entrada del esófago se consigue mediante el desplazamiento anterior de la laringe y la relajación del EES, compuesto por el músculo constrictor inferior de la faringe y el músculo cricofaríngeo, el cual es el principal responsable del tono del EES. ${ }^{3}$ Este esfínter se mantiene tónico durante la respiración para evitar el paso de aire al esófago, y se relaja durante la deglución, permitiendo el paso del bolo alimentario.

Cuando existe un fallo en la relajación de EES, se produce regurgitación oral y nasal, así como aspiración traqueobronquial. ${ }^{4}$ El trastorno de la relajación del cricofaríngeo durante el proceso de deglución en ausencia de otra anomalía neurológica o motora se denomina: acalasia cricofaríngea primaria. ${ }^{2}$

La acalasia cricofaríngea (AC) es una causa rara de disfagia. Fue descrita en 1925 por Jackson en adultos, y en 1969 por Thomas y Utian en la infancia. La poca información en pediatría sugiere una relación $\mathrm{H}: \mathrm{M}$ de $1: 1{ }^{5} \mathrm{Su}$ etiología no está bien definida y se relaciona con alteraciones de los plexos nerviosos intramurales, trastornos del sistema nervioso central e infecciones. La mayoría de las investigaciones abogan por un origen multifactorial. ${ }^{6}$

La AC puede desarrollarse antes de los seis meses de edad; sin embargo, el diagnóstico suele retrasarse, dada la falta de especificidad de sus síntomas, que incluyen atragantamientos, regurgitación, reflujo nasal, tos, cianosis, neumonía recurrente o fallo de medro, entre otros. ${ }^{4} \mathrm{El}$ proceso diagnóstico obliga a descartar otras malformaciones congénitas, como atresia esofágica, fístula traqueoesofágica o la hendidura laríngea. Una vez descartadas, el índice de sospecha de una AC debe ser alto, sobre todo en ausencia de otros trastornos acompañantes, ${ }^{2}$ para conseguir un diagnóstico precoz y la prevención de sus complicaciones. ${ }^{6}$

\section{PRESENTACIÓN DEL CASO}

Lactante femenino de un mes de vida, quien es enviada al Complejo Hospitalario Universitario de Canarias, Tenerife por fallo de medro. En cuanto a sus antecedentes personales, fue una recién nacida de 38 semanas, con peso adecuado para la edad gestacional (3,030 gramos). Nació por cesárea por presentación transversa, con Apgar 3/6/9 que precisó reanimación perinatal con ventilación con presión positiva intermitente y presión positiva continua en las vías respiratorias. Ecografía transfontanelar postnatal normal. No se detectaron alteraciones en el tamiz metabólico y auditivo. Al momento del ingreso no había recibido vacunas. Su alimentación era con lactancia materna exclusiva.

Se refiere que, en el momento de alimentación, succiona con avidez, pero a los pocos minutos comienza a acumular la leche en la boca, le cuesta deglutirla y llega a expulsarla por la nariz, apareciendo posteriormente taquipnea y sudoración durante unos segundos. En ocasiones presenta accesos de tos durante la toma, y se calma cuando consigue tragar. Sin vómitos. Se realiza observación directa de la alimentación, confirmando la narración de los padres.

A la exploración física destaca la antropometría con un peso en percentil $2(2,835 \mathrm{~g})$, talla de $50 \mathrm{~cm}$ (percentil 9) y perímetro cefálico de $35 \mathrm{~cm}$ (percentil 15). Escaso panículo adiposo con hipotrofia muscular, sin otros datos anormales.

Dado los problemas durante la alimentación, se decide colocar sonda nasogástrica (SNG) para asegurar la correcta alimentación y preservar la vía aérea. Los exámenes sanguíneos resultaron normales. Posteriormente, se inició estudio de disfagia con tránsito esofagogástrico (TEGD) y una ecografía abdominal. En el TEGD se visualiza una disminución del calibre a nivel de esófago superior con dilatación proximal, que condiciona regurgitación nasal significativa, así como paso importante de contraste a la vía respiratoria (Figura 1).

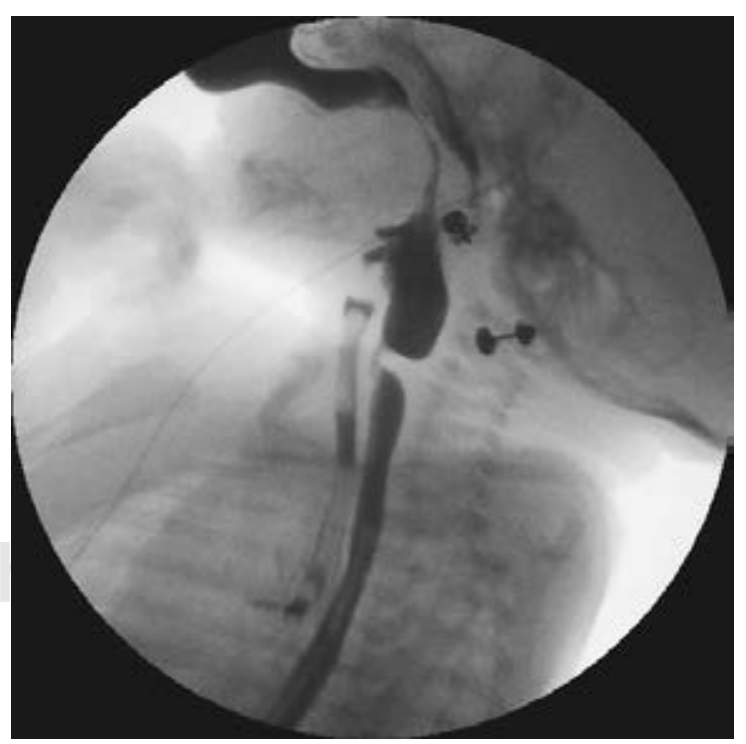

Figura 1: Disminución de calibre esofágico a nivel de C5-C6 con dilatación proximal, regurgitación nasal y paso de contraste a vía aérea. 


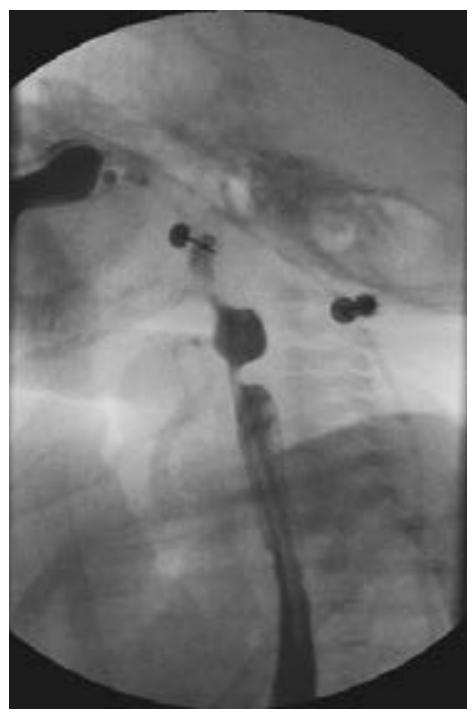

Figura 2: Persistencia de la acalasia cricofaríngea tras la segunda dilatación neumática.

Con el fin de descartar patología estructural o funcional se realizó resonancia magnética (RM) y tomografía computarizada (TC) cerebral y de fosa posterior, cervical y torácico. Además de fibroscopía para la exploración de vía aérea superior y tráquea. Así como endoscopía digestiva alta. Todo lo anterior resultó normal, por lo que estableció diagnóstico de acalasia cricofaríngea.

Tras obtener el diagnóstico, se consideró efectuar diagnóstico de certeza mediante manometría, lo cual no se realizó, dada la edad y peso de la paciente.

Como tratamiento inicial permanece con alimentación por SNG, con adecuada ganancia ponderal, así como tratamiento con omeprazol y antibioterapia empírica dada la broncoaspiración crónica. A los dos meses de vida, posterior a establecer el diagnóstico, se decidió realizar dilatación progresiva con balón en dos ocasiones (hasta 8 y $12 \mathrm{~mm}$, respectivamente), ambas bajo sedoanalgesia e intubación orotraqueal (IOT), transcurriendo las mismas sin incidencias. Se realizan esofagogramas tras ambas dilataciones, en los que se observó ausencia de paso de contraste a la vía aérea, pero persistencia de la imagen de estenosis a nivel del EES durante la ingesta, que disminuye en gran medida el calibre del esófago a ese nivel. Se decidió intervención quirúrgica por la persistencia de la anomalía (Figura 2).

A los cinco meses de vida, con un peso de 6,060 g (percentil 11), se efectúa cricomiotomía mediante cervicotomía lateral izquierda. En el sexto día postoperatorio se inicia tolerancia oral progresiva sin incidencias. En el decimoprimer día postoperatorio se consigue tolerancia oral completa y se realiza nuevo esofagograma, en el que ya no se aprecian estrechamientos en la luz del esófago (Figura 3).

A los tres meses de la intervención, la paciente había tenido adecuada ganancia pondero-estatural y apropiada ingesta por vía oral, sin atragantamiento o regurgitaciones.

\section{DISCUSIÓN}

La $\mathrm{AC}$ es un trastorno motor esofágico que se define como una disfunción del músculo cricofaríngeo y que consiste en la falta de relajación del mismo durante la deglución. Se trata de una entidad difícil de reconocer, pero se puede diagnosticar mediante un esofagograma o un TEGD, que muestra la impronta del músculo cricofaríngeo a nivel posterior de la faringe. ${ }^{6}$

Los hallazgos típicos en la AC son la acumulación de contraste en una faringe dilatada, con pequeño paso al esófago, una barra cricofaríngea o una protrusión posterior intermitente en la unión faringoesofágica a nivel de C5-C6, con regurgitación nasal o aspiración de contraste. ${ }^{2,4,7}$ Otros estudios complementarios, como la endoscopía superior, pueden ayudar a descartar enfermedad esofágica concomitante. ${ }^{3}$

El diagnóstico se confirma mediante manometría, pero no hay un patrón característico que permita, en todos los casos, el diagnóstico. ${ }^{6}$ Además, en niños pequeños resulta técnicamente difícil de realizarla, ya

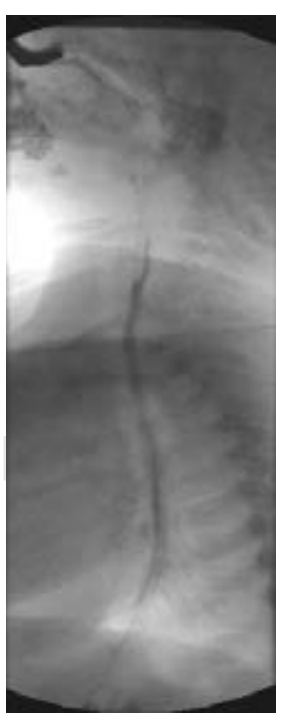

Figura 3: Resolución de la estenosis tras cricomiotomía. 
que en éstos la distancia entre la faringe y el EES es muy reducida y puede producirse desplazamiento de los transductores durante la deglución. ${ }^{4}$

En cuanto al tratamiento, en general, hay tres opciones. Dos médicas (dilatación con balón o inyección de toxina botulínica) y una quirúrgica (miotomía del cricofaríngeo). Sin embargo, hay diferentes posturas al respecto y no existe consenso. ${ }^{2,4,6,8}$ Existen corrientes que abogan por el uso de la dilatación endoscópica con aumento progresivo de la presión como primera opción terapéutica, dado que es una técnica menos invasiva y que hay evidencia de su efectividad hasta en $80 \%$ de los casos. ${ }^{2,3,8}$ Se debe tener en cuenta que, en ocasiones, será necesario repetir la dilatación varias veces para obtener resultados satisfactorios.

En cuanto a la inyección de toxina botulínica (2-7 $\mathrm{U} / \mathrm{kg}$ ), que se realiza en la pared anterior, ${ }^{3}$ su uso en la edad pediátrica no es frecuente, hay poca experiencia al respecto y no constituye una solución permanente. ${ }^{4,6}$

Por último, la miotomía del cricofaríngeo se considera la terapia quirúrgica de elección y definitiva. El índice de complicaciones es bajo y raramente son graves, por lo que se utiliza cuando no ha habido respuesta al tratamiento conservador. ${ }^{6}$ Por otro lado, el éxito de la intervención depende también de la asociación con otras anomalías esofágicas como el reflujo gastroesofágico. ${ }^{4,8}$

Por lo descrito, en la edad pediátrica el tratamiento de la AC no está claramente definido, pero en general, se aboga por el uso de la dilatación neumática como primera opción, reservando la cirugía y la toxina botulínica para los casos que no responden. En pacientes adultos, la cirugía es la primera línea de tratamiento, dada la eficacia de la técnica.

\section{REFERENCIAS}

1. García-Burriel JI. Disfagia en la infancia. An Pediatr Contin. 2014; 12(5): 221-230.

2. Akcaboy M, Zorlu P, Demir N, Acoglu EA, Oguz MM, Şenel S. A rare cause of dysphagia in children: primary cricopharyngeal achalasia. J Paediatr Child Health. 2017; 53(8): 827-828.

3. Carro GFM, López, CA, Gallardo OFJ, Pacheco RMA, Rivera MCG, Alfaro Ml et al. Miotomía quirúrgica del cricofaríngeo. An Orl Mex. 2016; 61(2): 146-153.

4. Drendel M, Carmel E, Kerimis $P$, Wolf $M$, Finkelstein $Y$. Cricopharyngeal achalasia in children: surgical and medical treatment. Isr Med Assoc J. 2013; 15(8): 430-433.

5. Gollu G, Demir N, Ates U, Aslan SS, Ergun E, Kucuk G et al. Effective management of cricopharyngeal achalasia in infants and children with dilatation alone. J Pediatr Surg. 2016; 51(11): 1751-1754.

6. Rodríguez-Iglesias $P$, Ibáñez-Pradas $V$, Alamar-Velázquez A, IbáñezAlcañiz I, Couselo-Jerez M. Cricopharyngeal achalasia: diagnosis and therapeutic alternatives. Cir Pediatr. 2015; 28(2): 81-83.

7. Morcillo-Carratalá R, Rodríguez-Laval V, Cruz-Hernández LM, Hernández-Guilabert PM, García-Sanz L. Esofagograma con bario: anatomía y patología básica. Radiología. 2014; 56 (Espec Cong): 164.

8. Marchica C, Zawawi F, Daniel SJ. Management of cricopharyngeal achalasia in an 8-month child using endoscopic cricopharyngeal myotomy. Int J Pediatr Otorhinolaryngol. 2017; 101: 137-140. 